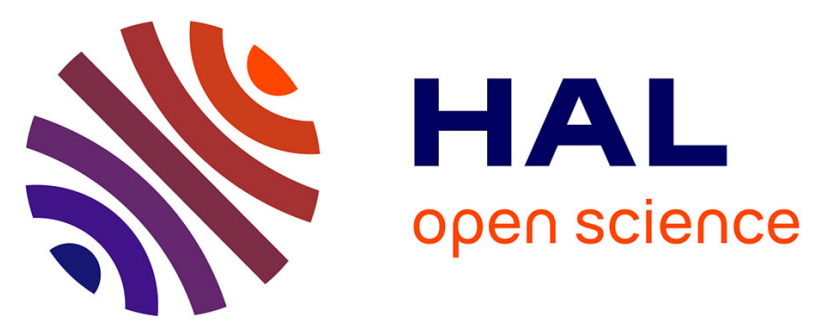

\title{
Comparison of Multi-resolution Analysis Patterns for Texture Classification of Breast Tumors Based On DCE-MRI
}

\author{
Alexia Tzalavra, Kalliopi Dalakleidi, Evangelia I. Zacharaki, Nikolaos \\ Tsiaparas, Fotios Constantinidis, Nikos Paragios, Konstantina S. Nikita
}

\section{To cite this version:}

Alexia Tzalavra, Kalliopi Dalakleidi, Evangelia I. Zacharaki, Nikolaos Tsiaparas, Fotios Constantinidis, et al.. Comparison of Multi-resolution Analysis Patterns for Texture Classification of Breast Tumors Based On DCE-MRI. MICCAI 2016 - 7th International Workshop on Machine Learning in Medical Imaging, Oct 2016, Athens, Greece. pp.296-304, 10.1007/978-3-319-47157-0_36 . hal-01359118

\section{HAL Id: hal-01359118 \\ https://hal.science/hal-01359118}

Submitted on 1 Sep 2016

HAL is a multi-disciplinary open access archive for the deposit and dissemination of scientific research documents, whether they are published or not. The documents may come from teaching and research institutions in France or abroad, or from public or private research centers.
L'archive ouverte pluridisciplinaire HAL, est destinée au dépôt et à la diffusion de documents scientifiques de niveau recherche, publiés ou non, émanant des établissements d'enseignement et de recherche français ou étrangers, des laboratoires publics ou privés. 


\title{
Comparison of Multi-resolution Analysis Patterns for Texture Classification of Breast Tumors Based On DCE- MRI
}

\author{
Alexia Tzalavra ${ }^{1}$, Kalliopi Dalakleidi ${ }^{1}$, Evangelia I. Zacharaki ${ }^{2}$, Nikolaos Tsiaparas ${ }^{1}$, \\ Fotios Constantinidis ${ }^{3}$, Nikos Paragios ${ }^{2}$ and Konstantina S.Nikita ${ }^{1}$ \\ ${ }^{1}$ School of Electrical and Computer Engineering, National Technical University of \\ Athens, Greece \\ \{atzalavra, kdalakeidi, ntsiapar, nkinita\}@biosim.ntua.gr \\ ${ }^{2}$ CentraleSupélec, Inria, Université Paris-Saclay, France \\ \{evangelia.zacharaki, nikos.paragios\}@centralesupelec.fr \\ ${ }^{3}$ NHS Greater Glasgow \& Clyde, Glasgow, UK \\ Fotios.Constantinidis@ggc.scot.nhs.uk
}

\begin{abstract}
Although Fourier and Wavelet Transform have been widely used for texture classification methods in medical images, the discrimination performance of FDCT has not been investigated so far in respect to breast cancer detection. In this paper, three multi-resolution transforms, namely the Discrete Wavelet Transform (DWT), the Stationary Wavelet Transform (SWT) and the Fast Discrete Curvelet Transform (FDCT) were comparatively assessed with respect to their ability to discriminate between malignant and benign breast tumors in Dynamic Contrast-Enhanced Magnetic Resonance Images (DCE-MRI). The mean and entropy of the detail sub-images for each decomposition scheme were used as texture features, which were subsequently fed as input into several classifiers. FDCT features fed to a Linear Discriminant Analysis (LDA) classifier produced the highest overall classification performance $(93,18 \%$ Accuracy).
\end{abstract}

Keywords: breast tumor diagnosis, DCE-MRI, texture, wavelet, classification

\section{Introduction}

Breast cancer is a primary cause of mortality and morbidity in women. It is commonly conceded that early diagnosis can be the key to increased survival rates and also to more specific and less aggressive therapy options. Breast magnetic resonance (MR) imaging has emerged as a promising modality for breast cancer detection [1]. Dynamic contrast-enhanced MR imaging (DCE-MRI) involves assessing the changes in signal intensity over time. This follows the intravenous injection of a paramagnetic contrast agent [2].

Several machine learning approaches have been proposed to analyze DCE-MRI data for breast tumor diagnosis. The implemented methods vary not only regarding the features extracted but also regarding the classification techniques used. A wide range of features have been explored in breast tumor Computer Aided Diagnosis (CAD) systems. Dynamic features $[3,4]$ have been used to characterize the temporal enhancement pattern of a tumor, while architectural features [3, 4] have been extracted 
to characterize the morphology of the tumor. Moreover, kinetic [5, 6] and texture features $[7,8]$ have been used to distinguish between malignant and benign tumors. More specifically, Yao et al. [8] computed textural features based on the cooccurrence matrix and also extracted frequency features by applying the discrete wavelet transform (DWT) on the texture temporal sequences of the breast tumors in order to classify them. Shannon et al. [9] applied textural kinetics to capture spatiotemporal changes in breast lesion texture in order to distinguish malignant from benign lesions. Furthermore, spatiotemporal features have proved to exhibit high performance in charactering breast tumors. Zheng et al. [10] used spatiotemporal enhancement patterns involving Fourier transformation and Gabor filters to analyze breast tumors. Gal et al. [11] extracted spatiotemporal features from a parametric model of contrast enhancement. Tzalavra et al.[12] extracted textural features from SWT detail sub-images in DCE-MRI data.

Furthermore, several classification methods have been used in breast tumor CAD systems. More specifically, Twellman et al. [13] presented a classification technique using artificial neural networks. Zheng et al. [10] assessed the diagnostic performance of the features they extracted for differentiating between benign and malignant tumors using linear discriminant analysis (LDA). Yao et al. [8] used support vector machines (SVM) for breast tumor classification.

The DWT has been widely used in several texture classification methods in medical images $[14,15]$ due to its multi-resolution characteristics. The Stationary Wavelet Transform (SWT), a modified time-invariant version of DWT, has been used in texture classification tasks [16]. The FDCT has been effectively used for characterizing carotid atherosclerotic plaque from B-mode ultrasound and discriminating between symptomatic and asymptomatic cases [17].

The purpose of this paper was to investigate the efficiency of multi-resolution wavelet methods to characterize the texture of breast tumors on DCE-MRI data. Three different decomposition schemes, namely the DWT, SWT and FDCT were implemented in order to characterize the spatial enhancement of the breast tissue. A set of classifiers were used for evaluating each decomposition scheme's ability to discriminate between benign and malignant tumors. More specifically, the following classifiers were compared in terms of classification accuracy: Bagging, K-means, Decision Table, Logistic Model Trees, Multilayer Perceptron, Naïve Bayes and LDA.

\section{Multi-resolution Image Analysis}

Images usually contain information at multiple resolutions. Therefore, multiresolution analysis has emerged as a useful framework for many image analysis tasks. The approach followed in this study, consists of the following main steps: tumor segmentation, normalization across subjects, feature extraction from the tumor region and tumor classification into malignant or benign. In this study, tumor segmentation was manually performed by an expert radiologist. The manually segmented breast tumors are first spatially normalized using Principal Component Analysis (PCA), as described in [10], in order to eliminate scale variations. Fourier transform is subsequently applied to capture the temporal enhancement properties, hence to kinetic in- 
formation. Then 3D wavelet transforms were applied to capture the spatiotemporal characteristics of the tumor. Specially, the FDCT method allows capturing both spatial and temporal characteristics, as described in section 3.2 below. Texture features from the resulting images were extracted and introduced into different classifiers for tumor classification.

Discrete Wavelet Transform. The two dimensional DWT is an effective tool to analyze images in a multi-scale framework [18]. The DWT is implemented via iterative linear filtering and critical down-sampling on the original image yielding three highfrequency directional sub-bands at each scale level and also one low-frequency subband usually known as image approximation. Directional sub-bands are sub-images exhibiting image details according to horizontal, vertical and diagonal orientations $[19,20]$.

Stationary Wavelet Transform. The SWT [21] is a translation-invariance modification of the DWT. More specifically, no down-sampling is performed in SWT. Instead, up-sampling of the low-pass and high-pass filters is carried out.

Fast Discrete Curvelet Transform. The FDCT [22, 23] involves initially the application of a 2D FFT to the image and then the windowing in a parallelogram of finite support for each scale and angle. The final result is obtained with the application of the 2D inverse FFT. FDCT [22] is more fast and accurate and less redundant than Discrete Curvelet Transforms (DCT).

\section{Materials and Methods}

\subsection{DCE MRI data}

The images used in this study were provided by the University of Pennsylvania. They were acquired from patients with breast tumors in a $1.5 \mathrm{~T}$ scanner (Siemens Sonata) or a $3 \mathrm{~T}$ scanner (Siemens Trio). In total, there were 44 subjects used, including 23 malignant and 21 benign cases. All of the samples were histologically verified. The boundary of the suspicious tumors was outlined on the images by a radiologist with expertise in breast imaging. Examples of benign and malignant tumors are shown in Fig. 1.
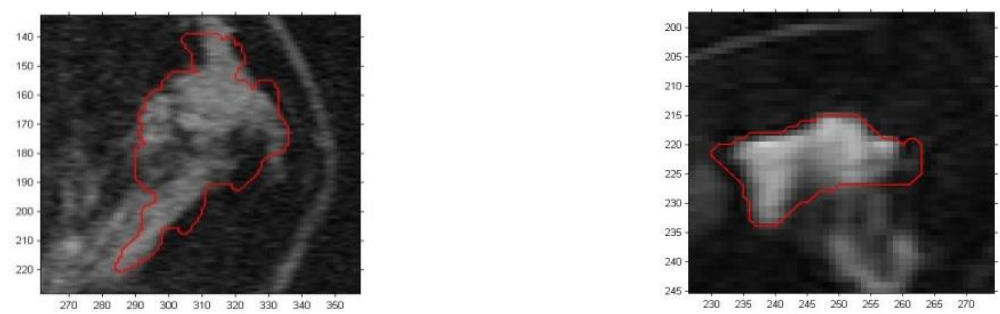

Fig. 1. Examples of a manually segmented malignant (right) and a benign (left) tumor 


\subsection{Extraction of texture features}

This section briefly describes the extraction of the texture features for each of the decomposition schemes.

The maximum value of decomposition of each of the investigated schemes equals to $\min \left(\log _{2} N, \log _{2} M\right)$, where $\mathrm{N}$ is the number of rows and $\mathrm{M}$ is the number of columns of the image. In our experiments $\mathrm{N}=\mathrm{M}=150$, thus the maximum level of decomposition equals to 7 . The statistics estimated from each detail sub-image were the mean and entropy of the absolute value of the detail sub-images, which both commonly have been used as texture descriptors.
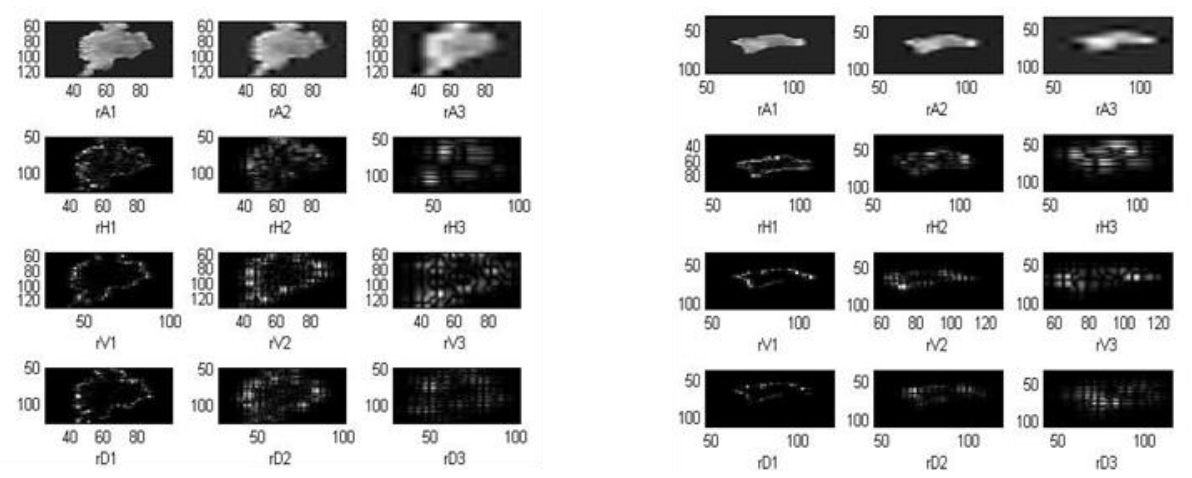

Fig.2 Examples of DWT sub images for 3 levels of decomposition for a malignant (right) and a benign (left) tumor (corresponding to images in Fig.1 (a) and (b)). The images in the first row correspond to the approximation images. For the images in rows $2-4$, each column corresponds to the detail sub-images of the levels 1-3 respectively
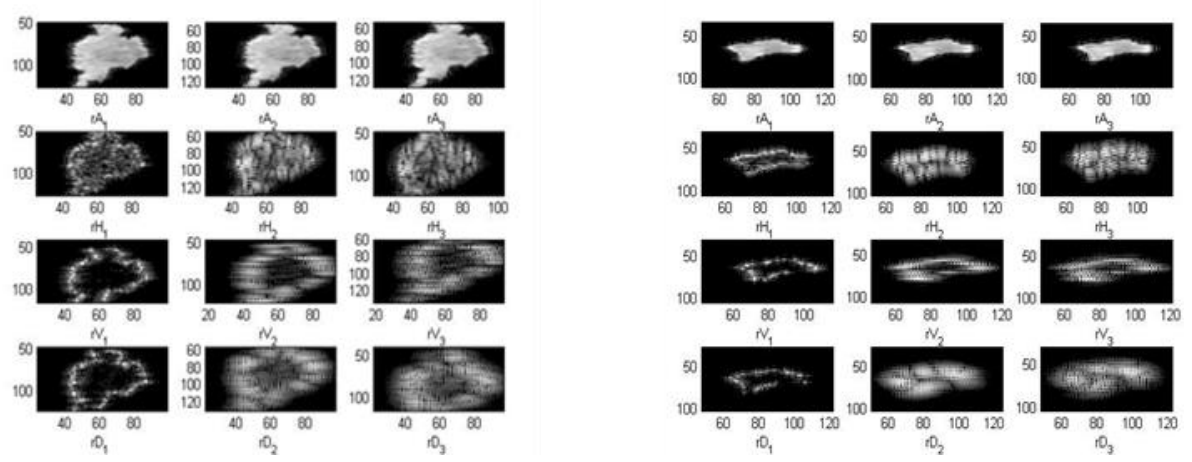

Fig.3 Examples of SWT sub images for 3 levels of decomposition for a malignant (right) and a benign (left) tumor (corresponding to images in Fig.1 (a) and (b)). The images in the first row correspond to the approximation images. For the images in rows $2-4$, each column corresponds to the detail sub-images of the levels 1-3 respectively

DWT and SWT: Several basic functions from different wavelet families were used, including Haar (haar), Daubechies (db), symlets (sym), coiflets (coif), and biorthogo- 
nal (bior). The 3-level decomposition scheme resulted in 9 detail sub-images for each time instance; hence totally 27 detail sub-images and consequently 54 texture features were obtained. The approximation sub-images were not used for texture analysis because they are the rough estimate of the original image. Fig. 2 and Fig.3 show examples of DWT and SWT detail sub-images.

FDCT: For the production of the detail sub images 4 decomposition scales were used. The number of angles for the second level was set to 16 (multiple of 4) and complex valued curvelets were used for the coefficients at the first level. For each level only the first half of the total coefficients was considered because curvelets produce symmetric coefficients for angles $\theta$ and $\theta+\pi$. The total number of curvelet coefficients obtained was 150 , leading to 300 texture features.

\subsection{Classification}

In order to classify the breast DCE-MRI tumors into benign and malignant, 6 classification algorithms in combination with 3 feature selection methods, provided by the WEKA 3 Data Mining Software [24], were used. The performance of these classifiers was compared with LDA. All classifiers were evaluated with the leave-one-out method.

Feature selection can be applied in two different ways, the wrapper approach and the filter approach. For the wrapper approach, two feature selection strategies were employed, the Best First (BF) [25] and the Simple Genetic Algorithm (SGA) [26], and were combined with the classifiers used later on for classification. For the filter approach, Information Gain (IG) [27] was used as the evaluation criterion of the features. The 10 best features according to the average value of information gain from the 44 leave-one-out iterations were then used for classification.

The following classifiers were used:

a) Bagging is a meta-classifier based on the bagging approach. The initial training set $D$ of size $N_{1}$ is used to generate $m$ new training sets $D_{i}$, each of size $\mathrm{N}_{2}$, by sampling from $\mathrm{D}$ uniformly and with replacement. The $\mathrm{m}$ base classifiers of the ensemble are trained with these $\mathrm{m}$ new training sets. Then, the $\mathrm{m}$ base classifiers are tested on a test set and their classification results are combined by voting.

b) K-means clustering [28] aims to partition $\mathrm{n}$ observations into $\mathrm{k}$ clusters in which each observation belongs to the cluster with the nearest mean.

c) A decision table majority classifier [29] consists of a schema which is a set of features that are included in the table and a body consisting of labeled instances from the space defined by the features in the schema. Given an unlabeled instance, a decision table classifier searches for exact matches in the decision table using only the features in the schema. If no instances are found the majority class of the classifier is returned, otherwise the majority class of all matching instances is returned.

d) Logistic Model Trees [30] are constructed by growing a standard classification tree, building logistic regression models for all nodes, pruning some of the sub trees using a pruning criterion, and then combining the logistic models along a path into a single model. 
e) Multilayer Perceptron is a neural network [31] with one or more hidden layers that uses back-propagation to estimate the weights of the network. All nodes of the network use the sigmoid transfer function.

f) Naïve Bayes [32] implements the probabilistic Naïve Bayes classifier, which is a specialized form of a Bayesian network, termed naïve because it relies on two important simplifying assumptions: firstly, that the predictive attributes are conditionally independent given the class, and, secondly that no hidden or latent attributes influence the prediction process.

g) Linear Discriminant Analysis classifier [33] is based on the fact that distributions, which have a greater variance between the two classes and smaller variance within each class, are easier to separate.

\section{Results}

Table 1 shows the classification results for all the above mentioned classifiers and all feature sets for each of the multi-resolution methods.

The highest accuracy and sensitivity scores for all methods are obtained with LDA. More specifically, for FDCT, LDA yielded an accuracy of $93.18 \%$ and a sensitivity of $100 \%$. Additionally, the meta-classifier based on K-means for the DWT and FDCT datasets yields the highest specificity value of $100 \%$.

Table 1. Classification results for multi-resolution schemes: DWT, SWT, FDCT: ACC: accuracy, SN: sensitivity, SP: specificity.

\begin{tabular}{|c|c|c|c|c|}
\hline \multirow{2}{*}{$\begin{array}{l}\begin{array}{l}\text { Multiresolution } \\
\text { Scheme }\end{array} \\
\text { DWT } \\
(\mathrm{db} 4, \mathrm{~L}=3)\end{array}$} & \multirow[b]{2}{*}{ Algorithm } & \multicolumn{3}{|c|}{ Classification performance $(\%)$} \\
\hline & & Accuracy & Sensitivity & Specificity \\
\hline & BF-Naïve Bayes & 84,09 & 73,91 & 95,24 \\
\hline & BF-Multilayer Perceptron & 77,27 & 69,57 & 85,71 \\
\hline & IG-Bagging & 79,54 & 78,26 & 80,95 \\
\hline & BF-K-means & 77,27 & 56,52 & 100,00 \\
\hline & BF-Decision Table & 72,73 & 69,57 & 76,19 \\
\hline & BF-Logistic Model Trees & 79,55 & 73,91 & 85,71 \\
\hline & LDA & 86,36 & 91,30 & 80,95 \\
\hline \multicolumn{5}{|l|}{$\begin{array}{l}\text { SWT } \\
(\operatorname{sym} 9, \mathrm{~L}=3)\end{array}$} \\
\hline & BF-Naive Bayes & 81,82 & 69,57 & 95,24 \\
\hline & BF-Multilayer Perceptron & 79,55 & 78,26 & 80,95 \\
\hline & SGA-Bagging & 79,55 & 73,91 & 85,71 \\
\hline & BF-K-means & 70,45 & 47,83 & 95,24 \\
\hline & BF-Decision Table & 86,36 & 78,26 & 95,24 \\
\hline & BF-Logistic Model Trees & 77,27 & 73,91 & 80,95 \\
\hline & LDA & 91,00 & $\mathbf{1 0 0 , 0 0}$ & 85,71 \\
\hline \multicolumn{5}{|l|}{$\begin{array}{l}\text { FDCT } \\
\text { (4 scales) }\end{array}$} \\
\hline & BF-Naive Bayes & 86,36 & 82,61 & 90,48 \\
\hline & BF-Multilayer Perceptron & 86,36 & 82,61 & 90,48 \\
\hline & Bagging & 77,27 & 73,91 & 80,95 \\
\hline & IG-K-means & 84,09 & 69,57 & 100 \\
\hline
\end{tabular}




\begin{tabular}{llll} 
BF-Decision Table & 81,82 & 78,26 & 85,71 \\
\hline IG-Logistic Model Trees & 81,82 & 78,26 & 85,71 \\
\hline LDA & $\mathbf{9 3 , 1 8}$ & $\mathbf{1 0 0 , 0 0}$ & 85,71
\end{tabular}

\section{Conclusion}

In this work, we investigated the possibility of using multi-resolution wavelet schemes to characterize the texture of breast tumors in DCE-MRI. Texture features were extracted from each scheme and fed into several classifiers. The experimental results illustrated high accuracy rates in breast tumor classification using FDCT and LDA as a classifier. Therefore, it can be concluded that curvelets can be key to breast tumor detection.

A main limitation of the method is its dependency on tumor boundary segmentation, currently performed manually. This limitation can be overcome by incorporating an automatic segmentation technique [34] making the method more robust and reproducible. Also, the refinement of the rough manual segmentation prior to feature extraction is possible to increase lesion classification accuracy, as shown in prior work [10]. Additional studies, systematically applying new multi-resolution schemes and more classifiers to larger populations, are expected to verify our findings. Finally, the use of automatic segmentation could result to ameliorated classification results.

\section{ACKNOWLEDGEMENTS}

The authors wish to thank Dr. Sarah Englander and Dr. Mitchell Schnall from University of Pennsylvania, USA, who supported the collection of the data. It should also be noted that K. V. Dalakleidi was supported by a scholarship for Ph.D. studies from the Hellenic State Scholarships Foundation "IKY fellowships of excellence for postgraduate studies in Greece-Siemens Program". This work has been partially supported from the European Research Council Grant 259112.

\section{References}

1. http://www.breastcancer.org/symptoms/understand bc/statistics.

2. S. G. Orel, M. D. Schnall, "MR imaging of the breast for the detection, diagnosis, and staging of breast cancer", Radiology, vol. 220, pp. 13-30, 2001

3. M. D. Schnall et al., "Diagnostic architectural and dynamic features at breast MR imaging: Multicenter study", Radiology, vol. 238, pp. 42-53, 2006

4. K. G. A. Gilhuijs et al., "Computerized analysis of breast lesions in three dimensions using dynamic magnetic-resonance imaging", Medical Physics, vol.25, pp. 1647-1654, 1998

5. W. Chen, M. L. Giger, U. Bick, G. M. Newstead, "Automatic identification and classification of characteristic kinetic curves of breast lesions on DCE-MRI", Med. Phys., vol. 33, pp. 1076-1082, 2006

6. S. H. Lee et al., "Optimal clustering of kinetic patterns on malignant breast lesions: comparison between K-means clustering and three-time-points method in dynamic contrast-enhanced MRI", Engineering in Medicine and Biology Society, 2007

7. P. Gibbs, L. W. Turnbull, "Textural analysis of contrast-enhanced MR images of the breast," Magn. Reson. Med., vol. 50, pp. 92-98, 2003

8. J. Yao, J. Chen, C. Chow, "Breast Tumor Analysis in Dynamic Contrast Enhanced MRI Using Texture Features and Wavelet Transform", IEEE Journal of selected topics in signal processing, vol. 3, no. 1,2009 
9. S. C. Agner et al., "Textural Kinetics: A Novel Dynamic Contrast-Enhanced (DCE)-MRI Feature for Breast Lesion Classification”, Journal of Digital Imaging, vol. 24, no. 3, pp. 446-463, 2010

10. Y. Zheng et al., "STEP: Spatiotemporal enhancement pattern for MR-based breast tumor diagnosis", Medical Physics, vol 36, no. 7, 2009

11. Y. Gal, A. Mehnert, A. Bradley, D. Kennedy, S. Crozier, "New Spatiotemporal Features for Improved Discrimination of Benign and Malignant Lesions in Dynamic Contrast-Enhanced Magnetic Resonance Imaging of the Breast”, Journal of Computer Assisted Tomography, vol. 35, no. 5, 2011

12. A. G. Tzalavra, E. I. Zacharaki, N. N. Tsiaparas, F. Constantinidis, K. S. Nikita, "A Multiresolution Analysis Framework For Breast Tumor Classification Based On DCE-MRI”, 2014 IEEE International Conference on Imaging Systems and Techniques (IST) Proceedings, pp. 246-250, 2014

13. T. Twellmann, O. Lichte, T. W. Nattkemper, "An adaptive tissue characterization network for modelfree visualization of dynamic contrast-enhanced magnetic resonance image data", IEEE Trans. Med. Imag., vol. 24, no. 10, pp. 1256-1266, 2005

14. M. Mojsilovic, M. V. Popovic, A. N. Neskovic, A. D. Popovic, "Wavelet image extension for analysis and classification of infracted myocardial tissue", IEEE Trans. Biomed. Eng., vol. 44, no. 9, pp. 856-866, 1997

15. D. R. Chen, R. F. Chang, W. J. Kuo, M. C. Chen, Y. L. Huang, "Diagnosis of breast tumors with sonographic texture analysis using wavelet transform and neural networks", Ultrasound Med. Biol., vol. 28 , no. 10 , pp. 1301-1310, 2002

16. N. N. Tsiaparas, S. Golemati, I. Andreadis, J. S. Stoitsis, I. Valavanis, K. S. Nikita, "Comparison of Multiresolution Features for Texture Classification of Carotid Atherosclerosis From B-Mode Ultrasound", IEEE Transactions on Information Technology in Biomedicine, vol.. 15, no. 11, 2011

17. N. N. Tsiaparas, S. Golemati, I. Andreadis, J. Stoitsis, I. Valavanis, K. S. Nikita, "Assessment of carotid atherosclerosis from B-mode ultrasound images using directional multiscale texture features", Measurement Science and Technology, vol. 23, no. 11, 2012

18. Mallat S. (1989) Theory for multiresolution signal decomposition: the wavelet representation. IEEE Transactions on Pattern Analysis and Machine Intelligence, 11/7:674-693

19. B. Furht, "Discrete Wavelet Transform (DWT)", Encyclopedia of Multimedia, Springer, USA, 2008.

20. http://users.rowan.edu/ polikar/WAVELETS/WTpart4.html, The Wavelet Tutorial, Part IV

21. B. S. Kumar, S. Nagaraj, "Discrete and Stationary Wavelet Decomposition for IMAGE Resolution Enhancement", International Journal of Engineering Trends and Technology (IJETT), vol. 4, no. 7, 2013

22. E. Candès, L. Demanet, D. Donoho, L. Ying, "Fast discrete curvelet transforms", Multiscale Modelling \& Simulation, vol. 5, no. 3, 2006

23. E. J. Candes, D. L. Donoho, "Curvelets, multiresolution representation, and scaling laws", SPIE Proceedings, vol. 4119, 2000

24. I.H. Witten, E. Frank, M. A. Hall, "Data Mining: Practical machine learning tools and techniques", Morgan Kaufmann, San Francisco, 2011

25. J. Pearl, "Heuristics: Intelligent Search Strategies for Computer Problem Solving", Addison-Wesley, 1984

26. D. Goldberg, "Genetic algorithms in search, optimization and machine learning", Addison-Wesley, Boston, MA, USA, 1989

27. C. D. Manning, P. Raghavan, H. Schuetze, “An introduction to Information Retrieval”, Cambridge University Press, 2008

28. J. A. Hartigan, “Clustering algorithms”, John Wiley \& Sons, Inc., 1975

29. R. Kohavi, "The Power of Decision Tables", in 8th European Conference on Machine Learn-ing, pp. 174-189, 1995

30. N. Landwehr, M. Hall, E. Frank "Logistic Model Trees”, Machine Learning, vol. 95, no. 1-2, pp. 161205, 2005

31. S. Haykin, "Neural Networks: A Comprehensive Foundation", Prentice Hall, 1999

32. G. H. John, P. Langley, "Estimating Continuous Distributions in Bayesian Classifiers", in Eleventh Conference on Uncertainty in Artificial Intelligence, San Mateo, pp. 338-345, 1995

33. P. A. Lachenbruch, Discriminant Analysis, Hafner, New York, 1975

34. Tianming Zhan, Renping Yu, Yu Zheng, Yongzhao Zhan, Liang Xiao, Zhihui Wei, "Multimodal spatial-based segmentation framework for white matter lesions in multi-sequence magnetic resonance images”, Biomedical Signal Processing and Control, volume 31, pp. 52-62, 2017 